\title{
Computer simulation of some of the motor circuits underlying Parkinson's disease
}

\author{
GABRIELLE GELLER and DAVID REYNOLDS \\ University of Windsor, Windsor, Ontario, Canada
}

\begin{abstract}
Many researchers have published functional models of brain structures involved in Parkinson's disease. These models include the primary structures of the basal ganglia and related cortical areas, and have hypothesized normal and dysfunctional states. Simple computer simulation of the hypothesized excitatory and inhibitory interactions among brain structures can be used to evaluate these models. A computer simulation can be programmed to simulate normal function, disease function, and treatment effects described in published models.
\end{abstract}

Parkinson's disease (PD) is a progressive disorder of the central nervous system characterized by four primary clinical features: rigidity, bradykinesia (slowness or lack of movement), tremor, and abnormal posture. When these symptoms predominate, get progressively worse, and there is no obvious cause, the patient is diagnosed with idiopathic Parkinson's disease (Clark, Brater, \& Johnson, 1992). The motor deficits of PD are accompanied by important pathological changes. There is substantial degeneration of pigmented neurons of the substantia nigra, pars compacta and parallel degeneration of dopaminergic neurons (Javitch, 1986). This degeneration has complex effects on the interconnections of the basal ganglia and cerebral cortex, resulting in the inhibition of voluntary movement and other motor and cognitive effects (Borison \& Diamond, 1987).

Parkinson's disease is the most studied and best understood of the movement disorders. An important step in clarifying the functional neuroanatomy of movement has been the elucidation of the parallel functioning of basal ganglia circuits (Alexander, DeLong, \& Strick, 1986). Five basal ganglia-thalamocortical loops have been identified. Each of these is functionally and structurally segregated (Alexander \& Crutcher, 1990), and each focuses on a particular region of the frontal cortex (Hoover \& Strick, 1993). All are involved in the generation and control of voluntary movement and include the skeletomotor, oculomotor, dorsolateral, prefrontal, lateral orbitofrontal, and anterior cingulate circuits (Alexander et al., 1986).

The skeletomotor or "motor" loop has been studied most extensively (Alexander \& Crutcher, 1990) and, because there are available models of its functioning under normal and abnormal conditions, it is the primary focus of this simulation. Many structures have been implicated in the neuropathology of the motor circuits underlying PD,

This paper is based on an earlier report submitted for partial fulfilment of a graduate course requirement. Correspondence should be addressed to G. Geller, Department of Psychology, University of Windsor, Windsor, ON, Canada N9B 3P4 (e-mail: geller@server.uwindsor.ca). the most frequently implicated of which include the $s u b$ stantia nigra, pars compacta (SNc), the putamen (Borison \& Diamond, 1987; Irwin, DeLanney, Forno, Finnegan, DiMonte, \& Langston, 1990); the globus pallidus, external segment (GPe; Albin, Young \& Penney, 1989); and the subthalamic nucleus (STN; Bergman, Wichmann, \& DeLong, 1990).

The output nuclei of the basal ganglia have also been implicated and include the globus pallidus, internal segment (GPi) and the substantia nigra, pars reticulata $(\mathrm{SNr})$; Albin, Young, \& Penney, 1989; Borison \& Diamond, 1987). In addition, this circuit involves the different nuclei of the thalamus, including the nucleus ventralis lateralis, pars oralis (VLo); the nucleus ventralis anterior, pars magnocellularis (VAmc); the nucleus ventralis anterior, pars parvocellularis (VApc); and the centromedian nucleus (CM; Alexander \& Crutcher, 1990). Different regions of the cerebral cortex implicated in the motor circuit include the supplementary motor area (SMA; Alexander et al., 1986); the premotor cortex (PMC; Alexander \& Crutcher, 1990; Hoover \& Strick, 1993); and the motor cortex (MC; Hoover \& Strick, 1993). Finally, a few studies have implicated the superior colliculus (SC; Albin et al., 1989; Graybiel, 1990), which is primarily involved in visual reflexes and reactions to moving stimuli.

Published models of PD not only identify the primary structures involved, but also provide a proposed system of excitatory and inhibitory connections and feedback loops in healthy and diseased systems. The inclusion of excitatory and inhibitory connections is based on the knowledge of the primary types of neurotransmitters and neuromodulators found at each connection. The primary neurotransmitters implicated in the motor circuit are dopamine (DA, with excitatory effects for D1 receptors and inhibitory effects for $\mathrm{D} 2$ receptors), gamma-aminobutyric acid (GABA, inhibitory effect), and glutamic acid or glutamate (GLU, excitatory effect; Graybiel, 1990).

The published models of the motor circuit range in complexity from relatively basic heuristic models (e.g., Bergman, Wichmann, \& DeLong, 1990) to more involved, 
complex models (e.g., Albin et al., 1989; Alexander et al., 1986). For the computer simulation, a basic model was used that can be conceptualized as a prototype of a single motor loop (Figure 1).

Under normal functioning, the model of the motor circuit has been hypothesized to work in the following manner: The cortex sends excitatory projections to the putamen and the subthalamic nucleus. The putamen also receives projections from $\mathrm{SNc}$. A "direct path" from the putamen to the output nuclei of the basal ganglia (GPi and $\mathrm{SNr}$ ) receives excitatory projections from $\mathrm{SNc}$ and, in turn, sends inhibitory projections to the output nuclei. At the same time, SNc sends inhibitory projections to the parts of the putamen involved in an "indirect path" to the output nuclei. These parts of the putamen then send inhibitory projections to GPe, which sends inhibitory projections to the subthalamic nucleus. The subthalamic nucleus completes the indirect loop by sending excitatory projections to GPi and SNr. Therefore, a "balanced opposition" (Bergman et al., 1990) occurs in this portion of the motor circuit that controls normal movement. The output nuclei send inhibitory projections to the superior colliculus and to nuclei of the thalamus. The thalamus

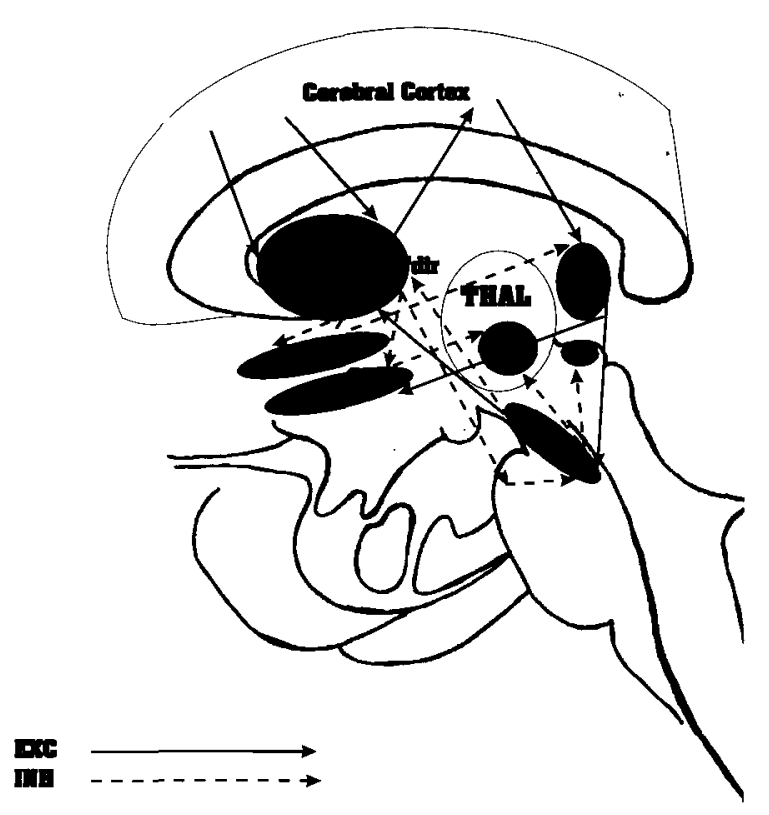

Figure 1. Basic model of the primary structures, and inhibitory and excitatory interconnections, of the motor circuit involved in the neuropathology of Parkinson's disease. GPi, internal segment of the globus pallidus; GPe, external segment of the globus pallidus; PUTdir, putamen direct route; PUTind, putamen indirect route; SC, superior colliculus; SNc, substantia nigra pars compacta; SNr, substantia nigra pars reticulata; STN, subthalamic nucleus; VLo, ventrolateral nucleus. Order of Connections for the Computer Simulation: (1) CC excites PUTdir; (2) CC excites PUTind; (3) CC excites STN; (4) SNc inhibits PUTind; (5) SNc excites PUTdir; (6) PUTind inhibits GPe; (7) GPe inhibits STN; (8) STN excites SNrGPi; (9) PUTdir inhibits SNrGPi; (10) SNrGPi inhibits SC; (11) SNrGPi inhibits VLo; (12) VLo excites CC. then sends excitatory projections back to various regions of the cerebral cortex, which completes the circuit (see Figure 1 for a schematic representation of normal function).

In a PD brain, the "balanced opposition" described is lost because of the destruction of nigrostriatal dopaminergic neurons and the resulting loss of DA. This loss affects the level of functioning of the structures involved in the circuit, resulting in decreased function of the cerebral cortex.

Computer simulations can be used to evaluate models of neuroanatomical function. The object-oriented approach (Booch, 1991) is well suited for simulation of neuroanatomical models because of the way it carries out simulations of complex systems. An object-oriented simulation works on the basis of the interactions of computational objects via a process called "message passing" (Booch, 1991). In addition, the encapsulation of the objects ensures that they are influenced only by selected projections from other objects. Encapsulation also ensures that different objects can respond in different ways to the same message (polymorphism). The simulation's ability to allow values within objects to change during a simulation (known as dynamic binding) is important for simulating ongoing dynamic interactions that occur in the brain. The purpose of the present study was to model neuroanatomical function of normal and PD states using object-oriented strategies.

Hypotheses related to treatment issues were tested upon the successful completion of the original model of PD functioning. At this time, the traditional treatments for PD include pharmacological treatments such as L-dopa therapy, specific D1 and D2 agonists, and stereotaxic interventions, involving lesions of the subthalamic nucleus and globus pallidus. The most common treatment uses L-dopa, a precursor of dopamine. L-dopa crosses the blood-brain barrier and results in an increase in production of dopamine. Unfortunately, side effects accompany the therapy, the most adverse being the development of dyskinesias, which are abnormal and excessive involuntary movements (Clark et al., 1992). Another problem with extended L-dopa therapy is that it loses its therapeutic effects after sustained use.

The limitations of L-dopa therapy have led researchers to study more specific dopamine agonists, including D1 and $\mathrm{D} 2$ receptor agonists. Although contradictory results as to which agonist is more effective have been reported (Boyce, Rupaniak, Stevenson, \& Iverson, 1990), there is general agreement that either agonist alone will not be completely effective because each works on only part of the motor circuit (Gerfen, 1992).

In experimental work on the potential therapeutic benefits of different lesions, two structures have frequently been studied, the subthalamic nucleus and the globus pallidus. Lesions of the subthalamic nucleus result in a decrease in the motor deficits of PD (Bergman et al., 1990; Hamada \& DeLong, 1992). However, it has also been postulated that lesions to the output nuclei of the basal ganglia (particularly GPi) would result in more beneficial 
effects than lesions to the subthalamic nucleus. This has been proposed because lesions to the subthalamic nucleus do not influence the direct route to the output nuclei (Contreras-Vidal \& Stelmach, 1995; Hoover \& Strick, 1993). This hypothesis has been partially supported in the literature (Baron, Vitek, Turner, Kareoke, Bakay, \& DeLong, 1993). These treatments were simulated using the computer model of PD and the hypotheses of the relative effectiveness of different treatments tested.

\section{Method}

Simulation software, written in the object-oriented language Smalltalk (Seguin, 1994), was designed to permit rapid prototyping of brain structure interaction. Brain structures and their excitatory and inhibitory projections were identified and entered into the simulation program. Normal brain structure activity was assigned an arbitrary value of 100 and remained at this level as long as no activity changes were introduced in any of the brain structures. The order of brain structure connections programmed closely paralleled the sequential components of the theoretical model (see Figure 1 for the order of connections). Brain systems were assumed to be in continuous activity and cycled through all connections in the order entered in the application software. Activity changes in any amount could be made in one or more brain structures. These changes were projected to subsequent brain structures and simply added or subtracted depending on whether these connections were excitatory or inhibitory. Once normal function was established, the output from the $\mathrm{SNc}$ was reduced to an output of 85 . The resulting changes in the structures' interconnections were analyzed and compared with changes hypothesized by the models. At this point, various treatments of PD (pharmacological and surgical techniques) were simulated and specific treatment hypotheses were tested.

\section{RESULTS}

\section{Validity Check}

The validity of the PD simulation was first established by determining the stability of the system when small fluctuations of different structures' output were manipulated (from 95 to 105 functioning). The validity check demonstrated that the model formed a relatively stable system. The manipulation of the cerebral cortex, for example, caused mild fluctuations in functioning of the other structures by 5 . Only two structures (the GPe and SC) fluctuated by 10 .

\section{PD Simulation}

To simulate the degeneration of dopaminergic nigrostriatal neurons, the output from the $\mathrm{SNc}$ was set at 85 . The hypothesized changes in the functioning of the other structures in the model were supported, as each structure in the simulation changed in the hypothesized direction, with the resulting cerebral cortex output decreasing (70). Other structures, such as putamen (direct route) and the $\mathrm{GPe}$, showed decreases in function (85). In addition, the putamen (indirect route) and the subthalamic nucleus increased in function (115). A larger increase in function was observed in the output nuclei of the basal ganglia (GPi and $\mathrm{SNr}$ to 130). The large increase in these structures' output led to a significant decrease in functioning of the VLo, the SC, and the CC (70).

\section{Treatment Simulations}

To simulate L-dopa therapy, the putamen direct route was manipulated to an increased function of 120 in order to mimic the effects of increased levels of DA reaching the D1 receptors of this structure. The putamen indirect route was also manipulated and set at 80 to mimic the effects of increased levels of DA reaching the D2 receptors of this structure. The resulting changes in function of the interconnected structures were in the hypothesized directions. The end result was an increase in function of the cerebral cortex from 70 to 110 .

To simulate D1 agonist treatment, the putamen direct route was manipulated to an increased function of 120 . This was done because this route is the primary D1 receptor pathway. As hypothesized, the end result was an increase in function of the cerebral cortex to 90 . The simulation also supported the hypothesized changes of the other structures involved.

To simulate D2 agonist treatment, the putamen indirect route was set at 80 functioning. This was done because this route is the primary D2 receptor pathway. As hypothesized, the end result was an increase in functioning of the cerebral cortex to 90 , the equivalent increase observed for D1 agonists. The simulation also supported the other hypothesized structure changes.

The final manipulations involved simulating therapeutic lesions. To mimic lesions of the subthalamic nucleus and $\mathrm{GPi} / \mathrm{SNr}$, these structures were set at 0 function. In addition, the programmed connections surrounding these structures were also removed. For the simulation of subthalamic lesions, this procedure resulted in an increase in function of the cerebral cortex to 85 . The same procedure was used to simulate globus pallidus lesions and resulted in an increase in function of the cerebral cortex to a normal 100 .

\section{DISCUSSION}

The hypothesized model of normal and PD neuroanatomical function was supported by the results of the computer simulation. In addition, the theoretically and empirically based hypotheses of proposed changes in structure functioning and relative effectiveness of various treatments were also supported by the simulation model. These findings not only lend support to published models of PD neuroanatomical function, but also demonstrate the utility of computer simulations as a strategy for evaluating models.

As predicted, when the degeneration of dopaminergic nigrostriatal neurons and the resulting depletion of dopamine was simulated, the remaining structures implicated in the motor circuit changed in the hypothesized directions. This resulted in significant decreased function of the cerebral cortex and the superior colliculus, which have both been associated with the motor deficits observed in PD patients.

In addition, when the computer model of $\mathrm{PD}$ function was "treated" with simulated L-dopa therapy, a signifi- 
cant increase in cerebral cortex function to above-normal levels was observed. Notably, Gerfen (1992) has proposed that overactivity in the cortex is the primary cause of the tolerance and dyskinesias observed with chronic L-dopa therapy. The results of the simulation also supported the hypothesis that although D1 and D2 agonists work on different portions of the motor circuit, they are equally effective. Also supported was the hypothesis that because of their selectivity, neither agonist alone is completely effective. Finally, the results of the simulation supported the empirically and theoretically based hypothesis that lesions to the output nuclei of the globus pallidus would be more effective in reducing motor deficits than would lesions to the subthalamic nucleus by increasing the function of the cerebral cortex. Although both types of simulated lesions increased function of the cerebral cortex and the superior colliculus, lesions to the output nuclei of the globus pallidus were more effective, returning function of these structures to 100 .

The simulation's ability to support the hypotheses derived from theoretical models provides support for both the model and the computer simulation. However, limitations are inherent in the present simulation software. For example, it does not allow differential weighting of projections. This is a limitation because in real brain function there are differences in the number and density of projections. A second limitation of the present software involves the necessity of entering the connections of structures in sequential order. The order in which these connections are entered can change the output of the simulation. This makes it difficult to simulate parallel activity in the system (i.e., one must use a sequential order of commands for processes that occur, at least in part, in parallel). Finally, the inability of the present computer program to simulate permanent damage is a stumbling block for those who wish to study the progressive effects of permanent damage through subsequent executions of the simulation.

Other limitations of the present study are inherent in the use of any simple model of neuroanatomical function to understand complex brain systems. Although the model is heuristically useful, it is by necessity far removed from the complex interconnectivity of most brain systems. For example, there is a multitude of intrinsic feedback connections from most brain structures that at this point cannot be modeled. However, despite these limitations, the general approach of dynamic, interacting brain structures is a promising advance over models based on "locality" assumptions (Farah, 1994).

The approach described here-linking human behavior to brain structure interaction-differs from conventional neural network approaches in terms of origin, empirical base, level of granularity, and goal. Neural networks originated in the mathematics of perceptrons (see Minsky \& Papert, 1969) and then evolved into connectionist networks (Rummelhart \& McClelland, 1986). In contrast, our simulations of brain-structure interaction derive from functional neuroanatomy (Nieuwenhuys, Voogd,
\& van Huijzen, 1988) and strategies implicit in objectoriented programming (Booch, 1991). One consequence of this difference in lineage is that the primitive units of neural networks are what Papert (1988) has called "mathematically purified" neurons, and the primitives of modular brain systems are more complex agents (Minsky, 1986), objects (Booch, 1991), or brain structures (Reynolds, Getty, \& Atwell, 1995).

In addition to differences in origin and level of granularity, the empirical base of neural networks has developed from attempts to answer Minsky and Papert's (1969) theoretical objections to early perceptrons with "new and improved" hidden-layer architecture and back propagation algorithms (Rummelhart \& McClelland, 1986). In contrast, brain-structure interaction models are based on large-scale neuroanatomical systems with specific excitatory and inhibitory pathways (Bressler, 1995; Koch \& Davis, 1994).

Finally, the major goal of the simulation approach described here is not to study the evolution (Reeke \& Sporns, 1993) or the training of neural networks (Rummelhart \& McClelland, 1986), but to model well-established, mature brain systems. In sum, the simulation of the largescale brain systems is different from (but does not reject) the neural network approach. An assumption basic to our approach is that fine-grain neurocomputation of neural networks may be appropriate for describing activity within brain structures. As reports of neural network computation within brain structures emerge, they can be embodied as encapsulated objects in large-scale simulations based on interactions among brain structures.

Modeling brain systems is a viable method of testing the validity and utility of various hypothesized models of neuroanatomical function. As computer software continues to evolve, simulations are becoming more sophisticated (see, e.g., Contreras-Vidal \& Selmach, 1995) and will be increasingly used to understand complex brain systems in PD and other brain diseases.

\section{REFERENCES}

Albin, R. L., Young, A. B., \& Penney, J. B. (1989). The functional anatomy of basal ganglia disorders. Trends in Neurosciences, 12, 366-375

Alexander, G., \& Crutcher, M. (1990). Functional architecture of basal ganglia circuits: Neural substrates of parallel processing. Trends in Neurosciences, 13, 266-271

Alexander, G., Delong, M., \& Strick, P. (1986). Parallel organization of functionally segregated circuits linking basal ganglia and cortex. Annual Review of Neuroscience, 9, 357-381.

Baron, M., Vitek, J., Turner, R., Kareoke, Y., Bakay, R., \& DeLONG, M. (1993). Lesions in the sensorimotor region of the internal segment of the globus pallidus (GPi) in Parkinsonian patients are effective in alleviating the cardinal signs of Parkinson's disease. Society for Neuroscience Abstracts, 15, 1584.

Bergman, H., Wichmann, T., \& DeLong, M. R. (1990). Reversal of experimental Parkinsonism by lesions of the subthalamic nucleus. Science, 249, 1436-1438.

Booch, G. (1991). Object-oriented design with applications. Redwood City, CA: Benjamin/Cummings.

Borison, R., \& Diamond, B. (1987). Neuropharmacology of the extrapyramidal system. Journal of Clinical Psychiatr, 48, 7-12.

Boyce, S., Rupaniak, N., Stevenson, M., \& Iverson, S. (1990). Dif- 
ferential effects of D1 and D2 agonists in MPTP-treated primates: Functional implications for Parkinson's disease. Neurology, 40, 927-933.

BRESSLER, S. (1995). Large-scale cortical networks and cognition. Brain Research Reviews, 20, 288-304.

Clark, W. G., Brater, D. C., \& Johnson, A. R. (1992). Drugs for treatment of movement disorders. In W. G. Clark, D. C. Brater, \& A. R. Johnson, Goth's Medical pharmacology (13th ed., pp. 286-299). St. Louis: Mosby.

Contreras-Vidal, J., \& Stelmach, G. (1995). A neural model of basal ganglia-thalamocortical relations in normal and parkinsonian movement. Biological Cybernetics, 73, 467-476.

FARAH, M. (1994). Neuropsychological inference with an interactive brain: Multiple levels of compartmental organization in the basal ganglia. Annual Review of Neuroscience, 15, 285-320.

GERFEN, C. R. (1992). The neostriatal mosaic: Multiple levels of compartmental organization in the basal ganglia. Annual Review of Neuroscience, 15, 285-320.

Graybiel, A. (1990). Neurotransmitters and neuromodulators in the basal ganglia. Trends in Neurosciences, 13, 244-253.

Hamada, L., \& DeLong, M. (1992). Excitotoxic acid lesions of the primate subthalamic nucleus result in reduced pallidal neuronal activity during active holding. Journal of Neurophysiology, 68, 1859. 1866

HoOver, J., \& STRICK, P. (1993). Multiple output channels in the basal ganglia. Science, 259, 819-821.

irwin, I., Delanney, L., Forno, L., Finnegan, K., DiMonte, D., \& LANGSTON, J. (1990). The evolution of nigrostriatal neurochemical changes in the MPTP-treated squirrel monkey. Brain Research, $\mathbf{5 3 1}$, 242-252.
JAVITCH, J. (1986). MPTP-induced neurotoxicity: Clues to the pathophysiology of Parkinson's disease. Integrative Psychiatry, 4, 81-88.

KoCH, C., \& DAVIS, J. (EDS.) (1994). Large-scale neuronal theories of the brain. Cambridge, MA: MIT Press.

Minsky, M. (1986). The society of mind. New York: Simon \& Schuster. Minsky, M., \& PAPERT, S. (1969). Perceptrons: An introduction to computational geometry. Cambridge, MA: MIT Press.

Nieuwenhuys, R., Voogd, J., \& van Huizzen, C. (1988). The human central nervous system. New York: Springer-Verlag.

PAPERT, S. (1988). One AI or many? In S. Graubard (Ed.), The artificial intelligence debate: False starts, real foundations (pp. 1-14). Cambridge, MA: MIT Press.

REEKE, G. N., \& SPORNS, O. (1993). Behaviorally based modeling and computational approaches to neuroscience. Annual Review of Neuroscience, 16, 597-623.

Reynolds, D. V., Getty, H. A., \& Atwell, G. (1995, June). Objectoriented computer models of brain disorders based on functional neuroanatomy. Paper presented at the Workshop on Neural Modeling of Cognitive and Brain Disorders, University of Maryland, College Park.

Rummelhart, D. E., \& McClelland, J. L. (1986). Parallel distributed processing: Explorations in the microstructure of cognition. Volume 1: Foundations. Cambridge, MA: MIT Press.

Seguin, M. (1994). Brain Structure Simulator [Computer software]. Windsor, ON, Canada: Author.

(Manuscript received September 30, 1996; revision accepted for publication January 14, 1997.) 